\title{
Résister ou collaborer? Étude socioprofessionnelle des magistrats « belges » et « français » sous le Directoire 1
}

\author{
Emmanuel Berger
}

Emmanuel Berger : Emmanuel Berger est docteur en histoire de l'Université catholique de Louvain et de l'Université Paris 1, il est actuellement Fellow au Netherlands Institute for Advanced Study (NIAS). Ses travaux portent sur l'histoire de la justice et des révoltes populaires dans l'Europe du $19^{\mathrm{e}}$ siècle. Il a notamment publié Popular Justice in Times of Transition (19th and 20th Century Europe) (Il Mulino/Duncker \& Humblot, 2017), Popular Justice in Europe (18 $8^{\text {th }}-19^{\text {th }}$ Centuries) (Il Mulino/Duncker \& Humblot, 2014), Popular Protest and Violence in 19 Century Europe (European Review of History, vol. 20, n6, 2013).

DOI: $10.25518 / 1370-2262.365$

\section{Résumé :}

À la suite l'annexion de la « Belgique » à la République française le $1^{\text {er }}$ octobre 1795, le Directoire décide d'exporter le modèle judiciaire de la Révolution dans nos régions. $\mathrm{Si}$, initialement, les nouveaux magistrats sont nommés par le gouvernement, ce dernier organise dès germinal an $\mathrm{V}$ des élections judiciaires. Pour la première fois, des juges élus localement sont chargés d'exécuter les lois adoptées à Paris. Notre article a pour objectif de croiser les identités socioprofessionnelles et les pratiques pénales des juges et des procureurs siégeant, sous le Directoire, au sein de l'arrondissement judiciaire. À partir de cette circonscription, nous serons à même de comprendre les différentes dynamiques de résistance et de collaborations des magistrats, qu'ils soient élus ou nommés, belges ou français.

Mots-clés : Directoire, Élection, Justice pénale, Magistrature

\begin{abstract}
:
Following the annexation of « Belgium » to the French Republic on October 1st, 1795, the Directory decides to export the judicial model of the Revolution in these regions. If initially the new magistrates are appointed by the government, the latter organizes judicial elections from Germinal Year V. For the first time, judges elected locally are responsible for enforcing laws voted in Paris. My article aims to cross the socio-professional identities and the penal practices of the judges and the prosecutors, under the Directory, within the judicial district. From this district, I shall be able to understand the various dynamics of resistance and collaborations of the magistrates, whether they are elected or appointed, Belgian or French.
\end{abstract}

Keywords : Criminal justice, Election, French Directory, Judiciary

L'histoire mouvementée de la justice belge à l'époque française (1795-1815) soulève de nombreux débats qui témoignent de l'ampleur des ruptures introduites par la Révolution. Les problématiques touchent, à la fois, à l'exportation du modèle judiciaire français, aux pratiques des nouvelles juridictions, à l'élection des magistrats, à l'établissement du jury populaire, etc. Le renversement de la justice d'Ancien Régime pose notamment la question des continuités et des ruptures juridiques, des résistances et des ralliements des magistrats à la République2. Face à ces interrogations, 
Résister ou collaborer ? Étude socioprofessionnelle des magistrats « belges »...

d'importantes avancées ont été réalisées au cours des vingt dernières années grâce, notamment, aux travaux d'historiens des lettres et d'historiens du droit tels que Xavier Rousseaux 3 , Marie-

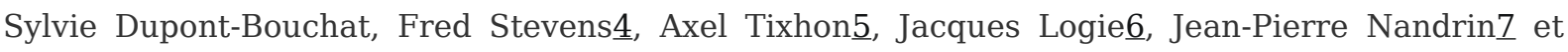
Emmanuel Berger. . À la suite de ces recherches, l'espace belge constitue aujourd'hui l'un des territoires européens dont l'histoire judiciaire est la mieux connue pour la période révolutionnaire et napoléonienne. L'intérêt porté à ce champ historiographique a également permis l'élaboration d'une base de données prosopographique couvrant l'ensemble de l'époque française. Elle a été conçue dans le cadre d'un projet plus large relatif à la «Prosopographie des magistrats belges (1795-1960) » . L'objectif du projet était d'offrir une vision à la fois synchronique et diachronique d'une juridiction, d'un magistrat ou de réseaux relationnels. Les données produites pour la période française ont principalement été recueillies à partir des notices rédigées par Jacques Logie10. L'outil informatique a permis de rationaliser les informations recueillies par ce dernier et d'opérer des requêtes sur les origines sociales, les carrières professionnelles, les orientations politiques ou les ressources financières des magistrats. Il ouvre la possibilité de croiser l'activité des tribunaux et le profil socioprofessionnel des magistrats qui les composent. Dans le cadre de cet article, nous nous concentrerons sur les identités et les pratiques pénales des juges et des procureurs siégeant, sous le Directoire (1795-1799), au sein d'une circonscription-clé créée par la Révolution : l'arrondissement judiciaire.

Suivant la Constitution de l'an III, l'arrondissement judiciaire est à la fois un lieu de poursuite des infractions et de répression des délits. C'est en effet dans son chef-lieu que se tiennent les séances du jury d'accusation et du tribunal correctionnel. Le jury d'accusation est chargé de déterminer s'il existe suffisamment d'indices de culpabilité pour renvoyer les prévenus devant le tribunal criminel du département. Le tribunal correctionnel est chargé, quant à lui, de juger les délits dont la peine n'est ni infamante ni afflictive, mais excède la valeur de trois journées de travail ou trois jours d'emprisonnement. Les peines d'emprisonnement ne peuvent dépasser deux années. En tant que pivot et relais de la dynamique judiciaire, l'arrondissement judiciaire apparaît, dès lors, comme l'une des pièces maîtresses de l'organisation pénale du Directoire11.

Le principal magistrat de l'arrondissement est le directeur du jury d'accusation. Ce dernier est désigné, tous les six mois, parmi les juges élus du tribunal civil du département. En tant qu'officier de police judiciaire, il dirige la poursuite de toutes les infractions conjointement avec les juges de paix dont il est le supérieur hiérarchique. Il dirige également les séances du jury d'accusation, réuni chaque décade, et préside le tribunal correctionnel aux côtés de deux juges de paix. Suivant les principes libéraux du modèle pénal de la Révolution, les pouvoirs du ministère public se limitent à requérir l'application de la loi et à exécuter les jugements. Contrairement aux directeurs du jury, les procureurs - appelés à l'époque commissaires du gouvernement - sont nommés et sont révocables par le Directoire. Si la limitation des prérogatives des commissaires était motivée par le souci de préserver l'indépendance de la justice face à l'influence du gouvernement, elle s'avère une source de conflits entre des juges indépendants conscients de leur légitimité populaire et des commissaires tenus d'appliquer les politiques pénales du Directoire $\underline{12}$.

Dans les départements belges, les tensions liées aux prérogatives des juges et des commissaires sont accrues par le rattachement récent de ces territoires à la République. La future Belgique est alors administrée par des Français dits « de l'intérieur » envoyés par Paris et perçus par conséquent comme «étrangers » par la population. Dans sa remarquable thèse, Jacques Logie a retracé la mise en place de la nouvelle organisation judiciaire et le processus de sélection des magistrats. 
Peu après l'annexion de la Belgique, le 9 vendémiaire an IV (1 ${ }^{\mathrm{er}}$ octobre 1795), les représentants du peuple Pérès et Portiez, auxquels succéda Bouteville, procédèrent aux premières nominations. La désignation des juges, à partir du 7 frimaire an IV (28 novembre 1795), était considérée comme une mesure transitoire dans l'attente des élections de germinal an V (mars-avril 1797).

\begin{tabular}{|c|c|c|c|c|}
\hline \multicolumn{5}{|c|}{ PROPORTION DES MAGISTRATS FRANÇAIS DANS LES 9 DÉPARTEMENTS BELGES } \\
\hline & \multicolumn{2}{|c|}{$\begin{array}{l}\text { Avant les premières élections judiciaires } \\
\text { de l'an V }\end{array}$} & \multicolumn{2}{|c|}{$\begin{array}{l}\text { À la fin du Directoire } \\
\text { (an VIII) }\end{array}$} \\
\hline & NB Français & \% Français & $\begin{array}{l}\text { NB } \\
\text { Français }\end{array}$ & $\begin{array}{l}\% \\
\text { Français }\end{array}$ \\
\hline Juges du tribunal civil (dpt.) & $43 / 186$ & $23 \%$ & $19 / 202$ & $9 \%$ \\
\hline $\begin{array}{l}\text { Présidents du tribunal } \\
\text { criminel (dpt.) }\end{array}$ & $5 / 9$ & $56 \%$ & $3 / 9$ & $33 \%$ \\
\hline Accusateurs publics (dpt.) & $7 / 9$ & $78 \%$ & $3 / 9$ & $33 \%$ \\
\hline Commissaires (dpt.) & $6 / 9$ & $67 \%$ & $8 / 9$ & $89 \%$ \\
\hline Substituts (dpt.) & $5 / 9$ & $56 \%$ & $4 / 9$ & $44 \%$ \\
\hline Commissaires (arr.) & $8 / 35$ & $23 \%$ & $12 / 35$ & $34 \%$ \\
\hline Total & $74 / 302$ & $24 \%$ & $49 / 273$ & $18 \%$ \\
\hline
\end{tabular}

À la veille des premières élections judiciaires de l'an V, la proportion de magistrats français est de vingt-quatre pourcents. Elle s'explique à la fois par la nécessité de combler les places laissées vacantes par les nombreux refus et démissions qui suivirent les premières nominations 13 et par la volonté politique de placer des Français «de l'intérieur» auprès de chaque juridiction. Les autorités se méfiaient, en effet, du conservatisme du monde judiciaire belge et incitèrent leurs compatriotes à accepter des places de juge dans les départements belges. Pour favoriser leur venue, le Directoire décida le 26 frimaire an IV (17 décembre 1795) d'allouer une avance de 10.000 livres en assignats à titre de frais de déplacement14. La nécessité d'avoir des hommes de confiance aux postes-clés explique la forte proportion de magistrats français (64\%) parmi les présidents et les accusateurs publics des tribunaux criminels, de même que parmi les commissaires et les substituts du département. Dans les juridictions théoriquement moins sensibles telles que celles d'arrondissement et les tribunaux civils, les Français ne constituent plus que vingt-trois pourcents des magistrats. À vrai dire, cette proportion rencontre les instructions antérieures du Comité de salut public invitant les représentants du peuple à ne pas dépasser le quorum de vingt-cinq pourcents de Français pour les nominations dans les administrations publiques $\underline{15}$.

La présence différenciée de Français, en fonction du type de juridictions et de magistrature, s'accentue à la fin du Directoire. À cette époque, les fonctions éligibles (juge du tribunal civil, président du tribunal criminel et accusateur public) ne sont plus occupées que par onze pourcents de Français «de l'intérieur». A contrario, le poids de ces derniers (45\%) est renforcé dans les places soumises à la nomination du gouvernement (commissaires et substituts). Cette évolution est visible au niveau de l'arrondissement où les Français représentent, en l'an VIII, trente-quatre pourcents des commissaires et à peine neuf pourcents des juges du tribunal civil d'où sont issus les directeurs du jury. L'importance de ce déséquilibre montre les limites des différentes législations qui autorisèrent le Directoire à contourner les élections et à nommer les juges. $\mathrm{Si}$, dans les départements belges, le gouvernement usa de cette prérogative pour nommer près du quart des magistrats, ces chiffres sont en réalité grossis par la situation particulière du département des 
Résister ou collaborer ? Étude socioprofessionnelle des magistrats « belges »...

Deux-Nèthes dans lequel tous les juges ont été nommés à la suite de l'annulation des élections de l'an V16. Les juridictions belges offrent, en définitive, un paysage contrasté, peuplé majoritairement de juges « locaux » tirant leur légitimité de l'élection et de commissaires du gouvernement, organes du nouveau régime, dont une part importante est originaire de la France métropolitaine. Il reste à déterminer quels furent les effets et les conséquences de ce déséquilibre tant dans l'organisation que dans les pratiques des juridictions pénales d'arrondissement. Pour ce faire, nous étudierons un échantillon de dix arrondissements dont les archives ont été particulièrement bien conservées pour la période directoriale 17.

PRÉSENCE DES MAGISTRATS FRANÇAIS PENDANT LE DiRECTOIRE (DU 7 FRIMAIRE AN IV AU 18 BRUMAIRE AN VIII (47 MOIS) $) \underline{18}$

\begin{tabular}{|l|l|l|l|l|l|}
\hline DÉPARTEMENTS & ARRONDISSEMENTS & \multicolumn{2}{l|}{$\begin{array}{l}\text { DIRECTEURS } \\
\text { JURY }\end{array}$} & \multicolumn{2}{l|}{ COMMISSAIRES } \\
\hline & & NB & Durée & NB & Durée \\
\hline DYLE & Bruxelles intérieur & 1 & 2 mois & 0 & 0 \\
\hline & Nivelles & 1 & 6 mois & 3 & 29 mois \\
\hline JEMAPPES & Mons & 0 & 0 & 2 & 14 mois \\
\hline DEUX-NĖTHES & Malines & 6 & 21 mois & 0 & 0 \\
\hline & Turnhout & 3 & 11 mois & 2 & 21 mois \\
\hline ESCAUT & Anvers & 2 & 2 mois & 1 & 24 mois \\
\hline LYS & Gand & 0 & 0 & 2 & 17 mois \\
\hline OURTHE & Bruges & 3 & 16 mois & 1 & 1 mois \\
\hline TOTAL & Waremme & 0 & 0 & 0 & 0 \\
\hline & Liège & 0 & 0 & 0 & 0 \\
\hline
\end{tabular}

Sur l'ensemble des arrondissements, la proportion de Français « de l'intérieur » parmi les directeurs du jury (17\%)19 et les commissaires du gouvernement (37\%)무 correspond à celle enregistrée par Jacques Logie. Les situations varient cependant fortement d'un arrondissement à l'autre. Certains (Waremme et Liège) n'ont accueilli aucun magistrat français tandis que d'autres (Nivelles et Turnhout) témoignent d’une présence métropolitaine plus marquée. Cette présence varie, en réalité, fortement suivant le type de magistrats. À l'image des résultats obtenus précédemment, les Français sont bien représentés parmi les commissaires du gouvernement. À Nivelles, Turnhout et Anvers, ils occupent leur fonction pendant la majeure partie du Directoire; à Gand et à Mons pendant une partie significative du régime. En ce qui concerne les directeurs du jury, la situation est inversée. À l'exception de Malines, Bruges et Turnhout, les juges français du tribunal départemental n’ont jamais exercé la fonction semestrielle de directeur du jury.

Ce constat s'explique tout d'abord par le nombre extrêmement réduit de juges originaires « de l'intérieur ». À la veille du Consulat, ces derniers siègent de manière tangible uniquement dans les départements des Deux-Nèthes (cinq), de la Lys (quatre) et des Forêts (quatre)21. Ce poids relatif pourrait expliquer la proportion plus importante de directeurs du jury français à Bruges (Lys), Malines (Deux-Nèthes) et Turnhout (Deux-Nèthes). La désaffection des juges métropolitains s'explique également par des facteurs communs à l'ensemble des magistrats belges. En effet, de manière générale, les fonctions de directeur du jury ne suscitaient guère d'enthousiasme. Outre la lourdeur de la charge de travail (instruction des infractions commises dans l'arrondissement, 
jugement des délits correctionnels et réunion du jury d’accusation), les juges désignés comme directeurs du jury étaient contraints de quitter le chef-lieu du département pour résider pendant six mois dans celui de l'arrondissement. Les déplacements, parfois longs, avaient pour conséquence de séparer les familles. Ils posaient également des problèmes matériels dans la mesure où les juges devaient assumer les frais de logement22. Enfin, dans les arrondissements néerlandophones ou germanophones, l'envoi de juges français ne connaissant pas suffisamment la langue locale pouvait être inapproprié.

Il est cependant intéressant de remarquer que de tels écueils ne semblent pas avoir découragé tous les magistrats « de l'intérieur ». Ce fut notamment le cas du Genevois Jean Tourn qui fut employé au district de Douai pendant la Révolution. Après avoir occupé les fonctions de chef de bureau à l'administration centrale et supérieure de Belgique puis au Conseil de gouvernement, il fut nommé juge au tribunal du département des Deux-Nèthes le 7 frimaire an IV (28 novembre 1795) 23. Réélu jusqu'à la fin du Directoire, il exerça à deux reprises les fonctions de directeur du jury de l'arrondissement de Turnhout (de prairial à messidor an V puis de brumaire à floréal an VI ; soit de mai à juin 1797, puis d'octobre 1797 à avril 1798). Si Jean Tourn possédait sans doute quelques notions de flamand avant de se rendre en Belgique, les conditions d'exercice de ses fonctions dans la petite ville de Turnhout n'en restent pas moins énigmatiques. Le cas de Tourn n'est pas unique. Si l'on excepte François Louis Huttin, Desmyter24 et Jean-Louis Carlier originaires de Dunkerque, les six autres directeurs de jury français proviennent de régions sans liens apparents avec les arrondissements belges : le créole Guillaume Filleul, natif de Saint-Domingue, est directeur du jury de Bruges entre floréal an VII et brumaire an VIII (avril et octobre 1799) ou encore Jacques Camus, originaire de Metz, siège à Malines entre messidor an IV et floréal an V (juin 1796 et avril 1797)25.

Lorsque l'on analyse l'origine géographique des commissaires du gouvernement issus «de l'intérieur », l'hétérogénéité constatée pour les juges disparaît : huit des onze commissaires sont natifs du département du Nord26. Le ministère de la Justice a, par conséquent, volontairement privilégié la nomination d'individus issus des territoires frontaliers. Il s'agit d'une politique constante puisque les nominations de «nordistes » se font tout au long du Directoire. Ce choix s'explique en partie par une plus grande facilité de recruter dans ces territoires des commissaires acceptant de se rendre dans les départements belges. Mais le principal motif réside dans l'obligation de disposer d'un personnel suffisamment familier de la région où il exerce ses fonctions. Une connaissance minimale des langues et des usages locaux s'avérait en effet indispensable pour contrôler l'activité pénale des juridictions. Si le gouvernement était favorable à une intégration des commissaires au sein des populations, une trop grande proximité pouvait toutefois s'avérer compromettante. Jean-Antoine Chaix, natif de Genève et commissaire près l'arrondissement de Mons, l'apprit à ses dépens. Nommé en brumaire an VI (octobre 1797), il fut révoqué le 29 nivôse an VII (18 janvier 1799) comme suspect de " protéger la caste nobiliaire, fréquenter leurs maisons et visiter dans la maison d’arrêt les prêtres condamnés à la déportation »27.

La question de l'intégration des magistrats au sein de leur arrondissement est fondamentale afin d'étudier l'impact des politiques répressives adoptées à Paris. À la fin du Directoire, quatre-vingtsix pourcents28 des juges belges des tribunaux civils résidaient dans le département avant leur

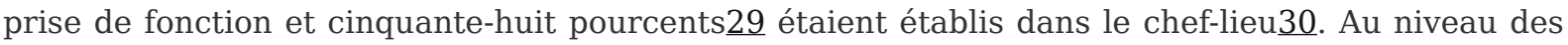
arrondissements étudiés, nous obtenons des résultats similaires : quatre-vingt-trois pourcents $\underline{31}$ des directeurs du jury résidaient dans le département de l'arrondissement où ils ont été désignés et soixante-quatre pourcents $\underline{32}$ dans le chef-lieu de leur juridiction. Toutefois, des variations importantes apparaissent suivant l'arrondissement. À Bruxelles, Nivelles, Mons, Gand et Liège, 
Résister ou collaborer ? Étude socioprofessionnelle des magistrats « belges »...

la majorité des directeurs du jury (86\%) résidaient dans le chef-lieu33. À Malines et Anvers, seule la moitié des magistrats y vivait $\underline{4}$. Enfin, aucun juge n'était originaire de Turnhout et de Waremme 35 . Ces variations s'expliquent par plusieurs facteurs. Tout d'abord, la plupart des chefslieux des arrondissements étudiés étaient également ceux des départements. Il s'ensuit que les juges qui y résidaient, étaient plus nombreux que dans les autres arrondissements. A contrario, il était plus difficile de trouver des magistrats dans les arrondissements ruraux moins densément peuplés ou sans tradition juridique forte tels que Waremme et Turnhout. La présence limitée de directeurs originaires d'Anvers et de Malines s'explique par l'annulation des élections de germinal an V (mars-avril 1797) et les difficultés subséquentes rencontrées par le Directoire pour constituer le tribunal du département吕.

Malgré l'ingérence du gouvernement dans les élections judiciaires et la désignation de magistrats français, les directeurs du jury sont majoritairement originaires des arrondissements où ils exercent leurs fonctions et par conséquent relativement proches des justiciables. Cette tendance lourde est d'autant plus intéressante que le ministre de la Justice Genissieu avait explicitement interdit le 6 ventôse an IV (25 février 1796) que les directeurs soient trop intégrés aux populations locales : «je suis informé que ces règles, prescrites aux tribunaux par la volonté du peuple français, sont violées sous vos yeux; je sais que des juges, contre l'ordre du tableau, ont été autorisés à remplir les rigoureuses fonctions de directeurs du jury d'accusation, et de présidents des tribunaux correctionnels dans des communes où ils avaient leurs domiciles avant d'être élus juges, et même uniquement parce qu'ils y avaient leurs domiciles. Il en résulte qu'un juge ainsi placé au milieu de ses voisins, de ses amis, et peut-être de ses ennemis, nourri de préventions locales et pour ainsi dire domestiques, exposé sans cesse aux sollicitations, aux insinuations de personnes au milieu desquelles il a vécu habituellement, et qui ont le secret de ses pensées et de ses affections les plus intimes, est dans l'impuissance de tenir avec impartialité et d'une main ferme la balance de la justice $\gg \underline{37}$.

Lorsque Charles Lambrechts est nommé ministre de la Justice, les arrangements pris par rapport à l'ordre du tableau et à la désignation de directeurs de jury régnicoles n'ont visiblement pas cessé. Le 3 floréal an VI (22 avril 1798), le ministre rappelle la nécessité absolue de respecter «l'ordre invariable du tableau et en souligne les avantages : les fonctions de directeur de jury sont à la fois pénibles pour celui qui s'en trouve chargé, et redoutables pour les citoyens soumis à sa juridiction. L'intention des législateurs a donc été de rendre plus supportable le fardeau qu'ils imposaient aux juges, par le moyen d'une égale répartition ; de contrebalancer l'influence de ces fonctions importantes, en prenant soin d'en abréger la durée ; enfin, de garantir le magistrat de toute affection locale, en lui ôtant la faculté de choisir le lieu dans lequel il doit les exercer ». La circulaire de floréal ne semble cependant pas avoir produit l'effet voulu. Lambrechts condamne à nouveau le 16 fructidor an VI (2 septembre 1798) «les arrangements arbitraires de quelques tribunaux [produits par] l'intérêt personnel et quelquefois malheureusement l'esprit de corps ».

Si le gouvernement condamne «l'esprit de corps » des tribunaux civils qui permet aux directeurs du jury de siéger dans l'arrondissement de leur résidence, il n'hésite pourtant pas à opérer de manière analogue avec ses propres représentants. À la fin du Directoire, quatre-vingt-trois pourcents $\underline{38}$ des commissaires belges près les tribunaux correctionnels sont nommés dans leur département de naissance et cinquante-sept pourcents $\underline{39}$ dans le chef-lieu de leur arrondissement. Ces pourcentages, semblables à ceux obtenus pour les directeurs du jury, seraient sans doute plus élevés si l'on disposait des données relatives aux lieux de résidence. En outre, contrairement aux fonctions temporaires des juges de l'arrondissement, celles des représentants du gouvernement se 
caractérisent par une relative stabilité. Ainsi, onze des trente-cinq commissaires près les tribunaux correctionnels restent en fonction tout au long du Directoire $\underline{40}$. Dans les départements de la Dyle et de l'Ourthe, la permanence du personnel est particulièrement forte puisqu'elle concerne respectivement quatre et trois arrondissements sur cinq. Si une telle stabilité peut s'expliquer à la fois par des motifs de fidélité politique et de compétence juridique, elle permet surtout au Directoire de disposer d'un moyen d'action efficace pour impulser les politiques pénales et assurer le maintien de l'ordre public au sein de l'arrondissement.

Afin de comprendre les différentes dynamiques entourant les relations entre les directeurs du jury et les commissaires du gouvernement, nous analyserons l'exécution, dans les départements belges, de l'une des législations pénales les plus controversées, à savoir celle relative à la police des cultes et aux serments des prêtres. La loi du 7 vendémiaire an IV (29 septembre 1795) exigeait que les ministres du culte reconnaissent que « l'universalité des citoyens français est le souverain et qu'ils promettent soumission et obéissance aux lois de la République ». Tout individu qui ne satisferait pas à la déclaration était punissable correctionnellement de 500 livres d'amende et de trois mois à un an d'emprisonnement. L'obligation de prêter serment rendit la loi extrêmement impopulaire et provoqua de nombreuses résistances tant parmi les prêtres que parmi les citoyens ordinaires. Le mouvement de désobéissance eut rapidement un retentissement national et créa de nombreuses tensions au sein de la magistrature.

Compte tenu du faible nombre de directeurs du jury français (17\%), il n'est pas aisé de comparer leur activité répressive à celle de leurs homologues belges. Sur la base des arrondissements retenus, les magistrats français ne semblent cependant pas se démarquer par un zèle excessif. Lorsque la loi du 7 vendémiaire an IV fut publiée entre les mois de floréal et de prairial an V (avril et mai 1797) suivant les départements, les premiers tribunaux correctionnels à poursuivre les prêtres, qui refusaient de faire la déclaration de soumission, furent ceux de Liège et de Bruxelles, tous deux présidés par des juges belges. À Liège, Jean Lemoine rendit le premier jugement le 25 floréal an V (14 mai 1797). Paul Delchef, curé de la paroisse de Saint-Nicolas-aux-mouches, fut condamné à trois mois d'emprisonnement et à 500 livres d'amende. Dans les jours suivants et tout au long de prairial (mai-juin 1797), le tribunal correctionnel de Liège prononça douze autres condamnations. À Bruxelles, Englebert Joseph Ippersiel prononça la première sentence le 3 prairial an V (22 mai 1797) à l'encontre du curé de l'hôpital Saint-Jean, Joseph François De Hase. Lors des séances des 6 et 7 prairial (25 et 26 mai 1797), le tribunal condamna cinq autres prêtres bruxellois. À la suite de sa condamnation, De Hase se porta en appel devant le tribunal criminel de la Dyle. Le 13 prairial ( $1^{\mathrm{er}}$ juin 1797), ce dernier acquitta le prêtre en invoquant le fait que la loi sur la police des cultes n'était pas constitutionnelle et exécutoire dans les départements belges. Le jugement provoqua un véritable séisme politique dans la mesure où les magistrats contestaient les prérogatives du Directoire en matière de publication des lois. Ils se permettaient, en outre, de suspendre l'exécution des lois - ce qui constituait un cas de forfaiture $\underline{41}$ - et de remettre en question la légitimité de la politique religieuse du Directoire. Sur le plan judiciaire, le jugement du tribunal criminel entraîna une paralysie générale des poursuites jusqu'au coup d’État du 18 fructidor an V (4 septembre 1797)느.

La polémique provoquée par l'exécution de la loi du 7 vendémiaire an IV trouve son origine dans l'activité répressive des présidents des tribunaux correctionnels de Liège et de Bruxelles, Lemoine et Ippersiel. Ces derniers n'étaient toutefois pas des jacobins enragés. Licencié en droit, Lemoine était membre de la municipalité de Herve en l'an III. Il fut nommé juge suppléant du tribunal civil 
Résister ou collaborer ? Étude socioprofessionnelle des magistrats « belges »...

de l'Ourthe le 27 frimaire an IV (18 décembre 1795) et siégea comme effectif jusqu'aux élections de l'an V. Réélu en germinal, Lemoine resta en fonction tout au long du Directoire. Nommé juge à Huy en brumaire an IX (octobre-novembre 1800) puis à Liège en nivôse (décembre 1800-janvier 1801), il mourut le 27 mars 180743. Ippersiel était, quant à lui, un ancien avocat au Conseil de Brabant qui devint chef de bureau à l'Administration Centrale et Supérieure de la Belgique. Nommé juge au tribunal de la Dyle le 28 frimaire an IV (19 décembre 1795), il fut réélu en l'an $\mathrm{V}$ et siégea, tout comme Lemoine, jusqu'à la fin du Directoire. Il poursuivit sa carrière jusqu'à son décès en 1830 en occupant successivement les fonctions de président du tribunal de Nivelles (an VIII), de Bruxelles (an VIII) et de conseiller à la Cour impériale de Bruxelles (1813)ㅆ44. Au vu de ces éléments biographiques, Lemoine et Ippersiel partagent plusieurs points communs : une collaboration précoce avec le régime français, une légitimité électorale en l'an $\mathrm{V}$ et une carrière professionnelle continue jusqu'à leur décès. Ils correspondent en réalité au profil des juges en exercice durant le Directoire : soixante-et-un pourcents des magistrats étaient issus du scrutin de l'an V et quatre-vingts pourcents étaient licenciés en droit. Si Lemoine et Ippersiel ont exercé une fonction administrative à l'époque de la deuxième occupation, leur élection en l'an $V$, de même que la continuation de leur carrière après la chute du Directoire, traduisent la modération de leurs opinions politiques $\underline{4}$.

L'initiative répressive des tribunaux liégeois et bruxellois tient en réalité moins au zèle des juges qu'au rythme avec lequel chaque administration départementale décida de publier la loi du 7 vendémiaire an IV. Les départements de l'Ourthe et de la Dyle furent les premiers à publier cette dernière respectivement les 29 germinal et $1^{\mathrm{er}}$ floréal an V (18 et 20 avril 1797). Le conflit soulevé peu de temps après, par l'affaire du curé De Hase, poussa les autres administrations départementales, de même que les municipalités, à surseoir à l'exécution de la législation dans l'attente que le Corps législatif se soit prononcé sur la suppression ou le maintien du serment. La quasi-absence de jugements correctionnels après le mois de prairial an V (mai-juin 1797) ne signifie cependant pas que les directeurs du jury renoncèrent à appliquer la loi. Susceptibles d'être accusés de forfaiture et soumis à d'intenses pressions de la part du ministre de la Justice, des commissaires du gouvernement et de l'accusateur public, plusieurs juges tentèrent de faire face à cette situation inédite. À Mons, le juge Nicolas Marie Joseph Perlau avait pris ses fonctions de directeur du jury en floréal, soit à l'époque de la publication de la loi du 7 vendémiaire an IV. Ancien avocat au Conseil du Hainaut, il avait été nommé commissaire national près le tribunal de district d'Ath en l'an III, puis juge au tribunal du département de Jemappes le 7 frimaire an IV (28 novembre 1795)ㅌ․ Il venait d'être élu en germinal an V (mars-avril 1797) lorsque les premières dénonciations à l'encontre des prêtres insermentés parvinrent aux juges de paix. Face au refus de ces derniers d'agir « sous prétexte du rapport éventuel de cette loi par le corps législatif », Perlau leur rappela «que nous ne sommes que les instruments passifs de la loi, qu'il ne nous appartient pas d'en examiner le fond et qu'il doit nous suffire qu'elles [les lois] soient revêtues extérieurement de toutes les formalités légales et publiés pour les faire exécuter » $\underline{4}$. Il énonçait en réalité l'un des principes fondamentaux de l'organisation judiciaire de la Révolution : le juge n'a pas à interpréter la légitimité de la loi mais doit, tel un automate, en assurer l'application immédiate. Conformément à son devoir, Perlau jugea le capucin Pierre-Joseph Rinchon le 9 fructidor an V (26 août 1797) et le condamna à trois mois de prison et 500 livres d'amende. Le jugement est néanmoins exceptionnel dans la mesure où, parmi les arrondissements étudiés, il s'agit de la seule sentence prononcée par un tribunal correctionnel avant le coup d'État du 18 fructidor (4 septembre 1797).

La correspondance entre les magistrats montre la position inconfortable de Perlau. Alors qu'il 
exhortait les juges de paix à activer les poursuites, ses efforts n'ont pas convaincu le commissaire près le tribunal civil du département, Simon Nazaire Chenard. Ce dernier considéra en effet à propos du jugement de Rinchon qu' « il est ridicule qu'on ait commencé à sévir contre un personnage aussi insignifiant lorsqu'on reste dans une inaction coupable par rapport aux abbés dont les délits sont

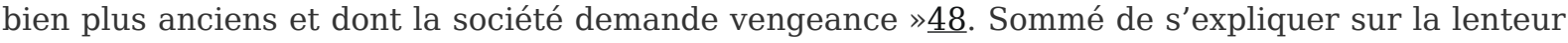
des poursuites, Perlau avait déjà fait part de son désarroi à l'accusateur public Joseph Giraud: «persuadez-vous que je ferai mon devoir, vous me connaissez et ne m'inculpez point de reproches (...). Tout cela est bien fort et trop fort pour moi. Plaignez-moi plutôt dans ces circonstances que de m'inculper de négligence $\gg \underline{49}$. Le découragement du directeur du jury s'explique par la nécessité de transiger avec la population montoise. Il assura à Giraud : « cela devient très sérieux, au point de compromettre (...) la sûreté et la tranquillité publique et particulièrement des agents du gouvernement $\gg \underline{50}$. Face aux pressions de sa hiérarchie et à l'impopularité de la loi, Perlau envisagea un instant de démissionner. Il resta cependant en fonction jusqu'à la fin de son semestre, en brumaire an VI (octobre-novembre 1797)토. Le malaise et l'impasse décrits par Perlau sont révélateurs de la position précaire du directeur du jury. Ce dernier était tenu d'exécuter une loi rejetée par la majorité des citoyens qui l'avaient élu. Lattitude de Perlau est caractéristique des républicains modérés, ralliés au nouveau régime mais attachés aux intérêts de la population locale. À l'image d'autres modérés tels que Lemoine et Ippersiel, il restera en place pendant tout le Directoire et siégera au tribunal de Mons jusqu'à sa mort en 1824.

Lactivité pénale décrite à Bruxelles, Liège et Mons est exceptionnelle. Dans les autres arrondissements, les tribunaux, pour la plupart présidés par des Belges, restèrent muets, attendant patiemment l'issue des débats au Corps législatif. L'étude de l'arrondissement de Malines tend à montrer que cette stratégie a également été suivie par les juges français. Le Douaisien Martin Louis Joseph Lebrête y occupa les fonctions de directeur du jury de prairial à thermidor an $\mathrm{V}$ (mai-juin 1797 à juillet-août 1797). Avocat d'opinion jacobine, il avait été chef de bureau au Conseil de gouvernement lors de la deuxième occupation. Nommé juge au tribunal des Deux-Nèthes le 7 frimaire an IV (28 novembre 1795), il fut réélu en germinal an V (mars-avril 1797) par l'assemblée scissionnaire dont les opérations ne furent finalement pas validées. Malgré cette déconvenue, le Directoire le nomma aux mêmes fonctions le 23 fructidor (9 septembre 1797). Les convictions politiques de Lebrête n'eurent guère d'influence sur l'activité pénale du tribunal correctionnel de Malines puisqu'aucun prêtre insermenté ne fut jugé pendant les mois litigieux. La première cause de cette inertie est, tout comme à Mons, à rechercher dans l'attitude des juges de paix.

Peu de temps après la publication de la loi du 7 vendémiaire par la municipalité de Malines, Lebrête enjoignit aux juges de paix de l'arrondissement de «donner aux lois précitées leur exécution». Il termina sa circulaire en tentant de se convaincre que «votre zèle pour la chose publique, l'attachement et l'activité au devoir que vous impose la loi me sont garants que vous ne négligerez rien» $\underline{52}$. Le doute exprimé à l'égard de l'enthousiasme des juges de paix à poursuivre se confirma rapidement. Lors de la séance du 14 prairial an V (2 juin 1797), le tribunal correctionnel malinois devait juger Vanbenghem, secrétaire de l'archevêque de Malines. Cependant, ce jour-là, Lebrête fut contraint de lire le courrier que lui avait adressé l'un des juges de paix composant le tribunal. Ce dernier déclarait qu'il ne pouvait siéger puisqu'il avait instruit l'affaire Vanbenghem et annonçait que ses six assesseurs avaient refusé de le remplacer. Trois lui avaient adressé leur démission, deux s'étaient portés malades et le sixième justifiait son absence par sa convocation comme juré d'accusation. Les différents désistements ne permettant pas de remplacer le juge de paix, les magistrats du tribunal se trouvèrent en nombre insuffisant et furent contraints de reporter 
Résister ou collaborer ? Étude socioprofessionnelle des magistrats « belges »...

le jugement de la cause. À vrai dire, la séance de mise en jugement intervenait au pire moment puisque dès le matin, la nouvelle de l'acquittement de De Hase, prononcé par le tribunal criminel de la Dyle, s'était répandue dans toute la ville. Les assesseurs des juges de paix n'allaient donc pas se risquer à juger un prêtre alors que la légitimité de la loi du 7 vendémiaire an IV était désormais remise en question. La paralysie du tribunal correctionnel provoqua la reprise des offices religieux qui s'étaient arrêtés depuis l'arrestation de Vanbenghem et, lorsque Lebrête osa évoquer la relance des poursuites, il essuya une démission en chaîne des commissaires de police, des juges de paix et de leurs assesseurs. Ces démissions se répercutèrent immédiatement sur l'activité judicaire, que ce soit en matière civile ou pénale.

Dans les courriers adressés au ministre de la Justice, Lebrête dénonça la "stagnation forcée » du tribunal provoquée par « les démissions ou les prétendues excuses (...) présentées au dernier moment ». Désormais, "le service public est mis dans un état qui prête à rire par une certaine classe de fanatiques qui suppose triompher ». Il relata surtout les événements et les rumeurs récents : le département de Sambre-et-Meuse a suspendu l'exécution de la loi, le tribunal criminel de la Dyle a libéré De Hase, la municipalité d'Anvers et plusieurs cantons de l'arrondissement de Malines ont refusé de publier la législation sur la police des cultes. Confronté à ces écueils, Lebrête assura Merlin de sa fermeté : «que fois-je faire ? Je ne dois que persister à maintenir l'exécution de la loi, jusqu'à ce qu'elle soit légalement rapportée et que ce rapport me soit connu officiellement $» \underline{53}$. Toutefois, trois semaines plus tard, l'assurance affichée par le directeur du jury ne résista pas à la réalité du terrain. Face au risque de paralysie de l'ensemble de l'appareil judiciaire, Lebrête décida, à l'instar des autres magistrats belges, de sursoir provisoirement aux poursuites des prêtres insermentés : « je m’aperçois que c'est un parti-pris, si je persiste dans ma réquisition commencée. Tous ces fonctionnaires publics sont décidés à donner leurs démissions et en ce cas, la justice civile et de conciliation va devenir nulle en ce canton, les justiciables vont languir et se plaindre (...). Cet alternatif est cruel pour moi malgré tout - j'ose dire - mon activité, mon désir et ma ferme résolution de donner à la loi existante toute son exécution. Dans l'état des choses, je me fais cette question. Quel est le plus prudent ou de paralyser entièrement le cours de toute justice ou de suspendre provisoirement l'un de ses effets! Je la vois aisée à résoudre. C'est de souffrir instamment qu'une branche souffre et faire en sorte d'alimenter le reste des rameaux et l'arbre qu'on peut conserver, du moins tant que le Corps législatif ait prononcé ou encore jusqu'à ce que le jugement du tribunal de la Dyle soit terminé en celui de cassation. Dans l'un et l'autre cas, je crois que le cours de la justice reprendra son activité qu'elle n'eût jamais dû quitter ; mais en attendant tout homme impatient conviendra que je suis à l'impossible d'aller plus avant, sans compromettre et la justice civile et la sûreté publique $» \underline{54}$.

Confrontés à l'inaction des tribunaux, les commissaires du gouvernement exprimèrent dans un premier temps leur consternation. À Bruxelles, apprenant le jugement du tribunal de la Dyle, Pierre-Joseph Vanderveken, originaire de Louvain, s'indigna : «quel scandale de voir fouler aux pieds les lois les plus sages sous prétexte que le Corps législatif n'en a point ordonné l'exécution dans ces départements " $\underline{5}$. Dans l'arrondissement de Mons, Pierre François Joseph Delneufcourt, natif de la même ville, critiqua « la motion plus qu'indiscrète du représentant Bonaventure $\underline{66}$ ?qui? a porté la plus grande atteinte au respect dû au gouvernement considéré ici tant sous le rapport de ceux à qui l'exécution des lois est confiée que relativement à la puissance de la loi $\gg \underline{57}$. À Malines, le Bruxellois François Joseph Auguste Sayavedra interpella directement le Directoire : « la marche de la justice s'est trouvée arrêtée par une manœuvre que tout semble attribuer à la malheureuse influence des prêtres et à la faiblesse des assesseurs de ce canton puisqu'il s'agissait 
de juger le nommé Charles-Antoine Depaule Vanbenghem se disant secrétaire de l'archevêque $» \underline{58}$. La réactivité et les propos engagés des trois magistrats belges s'expliquent sans doute en partie par leurs convictions politiques. Vanderveken et Delneufcourt avaient été vonckistes et étaient considérés sous le Directoire comme jacobins 59. Sayavedra avait, quant à lui, fait partie du Comité de surveillance de Bruxelles en l'an II et fut membre du tribunal criminel d'Anvers en l'an III $\underline{60}$.

En dépit de leur attachement à la République, les trois magistrats ne pouvaient toutefois pas faire abstraction des pressions locales et de l'incertitude juridique provoquée tant par le jugement du tribunal de la Dyle que par les recours législatifs. À la suite de l'annulation de la condamnation de De Hase, Vanderveken sembla moins pressé de poursuivre les contrevenants et demanda au ministre de la Justice s'il devait « continuer à traduire devant le tribunal ceux des prêtres qui sont en contravention à la loi?du 7 vendémiaire an IV? $» \underline{61}$. Sayavedra sollicita également Merlin afin de déterminer la marche à suivre pour remplacer les assesseurs démissionnaires. À Mons, Delneufcourt affirma que les directives ministérielles n'étaient pas nécessaires « pour animer mon zèle. Déjà le tribunal près lequel je suis a condamné Rinchon à trois mois de prison et 500 livres d'amende. Il poursuit les autres prêtres qui sont dans le cas de la loi. Il faut qu'aucune loi ne soit enfreinte dans une République $» \underline{62}$. Son enthousiasme passait cependant sous silence le fait que la condamnation de Pierre-Joseph Rinchon était l'unique sentence prononcée par le tribunal correctionnel de Mons. Le commissaire près le tribunal civil du département, Chenard, ne s'était d'ailleurs guère laissé impressionner par ce sursaut répressif et l'avait jugé « ridicule » $\underline{63}$.

Le comportement attentiste des commissaires du gouvernement belges ne se différencie toutefois pas de celui de leurs homologues français. Le commissaire près le tribunal correctionnel de Gand, Antoine Jean Bazire Lacoudraye, adressa le 23 thermidor an V (10 août 1797) à Merlin un rapport circonstancié et pessimiste sur l'état des poursuites contre les prêtres insermentés : " dans tout mon arrondissement, la loi du 7 vendémiaire est violée. Dans plusieurs cantons les commissaires près les administrations réclament contre cette violation. Dans tous, les juges de paix l'autorisent par leur inactivité. Quelques-uns rendent, en faveur des prêtres qui sont amenés devant eux, les ordonnances les plus injurieuses à la loi et les plus propres à aliéner tous les esprits et tous les cœurs. Même conduite est tenue par les prêtres et juges de paix des départements voisins » $\underline{64}$. Tout en dénonçant l'apathie des juges de paix, Lacoudraye admit lui-même, dans le même rapport, avoir requis la mise en liberté provisoire du curé de Notre-Dame de Gand. Le magistrat français reconnut en réalité s'être résigné à geler les poursuites : «j'aurais pu demander qu'il fût décerné un mandat d'amener contre chacun des prévenus. Ce parti aurait été le meilleur (...) mais étant assuré que le directeur du jury ?Charles Massez? n'accueillerait pas ma demande, j'ai craint en le forçant de se prononcer publiquement de procurer un nouvel avantage à ceux qui attaquent ouvertement la loi et d'encourager ceux qui n'ont pas encore osé la violer ». Les propos de Lacoudraye reflètent parfaitement les contraintes auxquelles étaient confrontés les commissaires, qu'ils soient belges ou français. Tenus de veiller à l'exécution de la loi, ces derniers étaient impuissants à influer sur la conduite des directeurs du jury à l'égard des prêtres insermentés. Charles Massez ne pouvait pourtant être soupçonné de cléricalisme. Ancien représentant provisoire de Gand en 1792 et propriétaire de biens nationaux, il était réputé d'opinion «démocrate » depuis la Révolution brabançonne65. L'attitude réservée des directeurs du jury les plus fidèles à la République avait également été relevée par Delfneucourt :« il y a dégoût dans les juges très patriotes qui tempèrent jusqu'à ce que le Corps législatif se soit prononcé dans un sens quelconque » $\underline{66}$.

Dans un contexte où la politique religieuse du Directoire n'a jamais été aussi impopulaire, la majorité des commissaires du gouvernement semble s'être accommodée de l'inertie des directeurs 
Résister ou collaborer ? Étude socioprofessionnelle des magistrats « belges »...

du jury. En quête de solutions, la mise en liberté provisoire des prêtres insermentés constituait, à leurs yeux, un compromis permettant de se concilier l'opinion publique de l'arrondissement tout en assurant le gouvernement de leur dévouement67. Cette stratégie fut également suivie dans les arrondissements de Bruxelles, de Mons et d'Alost68. Lors du coup d'État du 18 fructidor, l'entente affichée entre commissaires et directeurs du jury tout au long de l'an $\mathrm{V}$ se maintint mais changea de nature. Le coup d'État permit en effet au gouvernement de durcir le ton à l'égard de ses opposants. Le tribunal de cassation nouvellement épuré cassa le jugement du tribunal criminel de la Dyle69 et poursuivit trois juges de ce tribunal pour forfaiture. Sur le plan législatif, les deux assemblées également épurées mirent un terme aux débats sur la liberté religieuse. En matière de police des cultes, l'article 25 de loi du 19 fructidor an V (5 septembre 1797) conserva la déclaration exigée par la loi du 7 vendémiaire an IV mais en remplaça le contenu par un "serment de haine à la royauté et à l'anarchie, d'attachement et de fidélité à la République et à la Constitution de l'an Trois ». Désormais, la légitimité de la prestation de serment ne pouvait plus être contestée et les directives du ministre de la Justice indiquèrent clairement que le retard mis dans la poursuite des contrevenants n'était plus toléré. La surveillance accrue du gouvernement associée à la fin des incertitudes juridiques pesant sur la police des cultes incitèrent les directeurs du jury et les commissaires du gouvernement à juger les prêtres insermentés. Ce réveil répressif se traduisit par une augmentation rapide de l'activité des tribunaux correctionnels dès les premiers mois de l'an VI. Les juridictions correctionnelles traitèrent cinquante-quatre pourcents $\underline{70}$ des affaires de culte au cours de cette année, contre vingt-et-un pourcents 71 lors de l'année précédente 72.

Si l'accélération des poursuites est due en partie à la mise en jugement des affaires instruites en l'an $\mathrm{V}$ dont le renvoi devant la justice correctionnelle avait été temporairement reporté, elle s'explique surtout par les sanctions auxquelles s'exposaient les juges et les commissaires du gouvernement qui se montreraient récalcitrants. Les ministres de la Justice Merlin et Lambrechts rappelèrent en effet tout au long de leur correspondance l'application de l'article 26 de la loi du 18 fructidor an $\mathrm{V}$ qui prévoyait une peine de deux années de fers à l'encontre des magistrats qui n'exécuteraient pas ou entraveraient l'application de la législation sur la police des cultes. La menace de sanction ne parait cependant pas avoir sapé les relations solidaires établies entre les magistrats de l'arrondissement. Les rares cas de résistance aux directives ministérielles en attestent. Dans l'arrondissement d'Alost, le tribunal correctionnel osa acquitter, le 3 frimaire an VI (23 novembre 1797), le curé de la paroisse de Ressseghem, Vanransberghe, prévenu d'avoir célébré la messe, les $1^{\mathrm{er}}$ et $2^{\mathrm{ème}}$ jours complémentaires de l'an V (17 et 18 septembre 1797), sans avoir prêté le moindre serment. Le directeur du jury Jacques Liévin Joseph Vandeputte admit les arguments de la défense reposant sur le fait que les lois sur la police des cultes, n'ayant pas été publiées dans la commune, elles ne pouvaient y être appliquées.

Aux yeux du ministre de la Justice, il s'agit là d'un « ridicule prétexte $» \underline{73}$. Lambrechts demanda par conséquent au commissaire près le tribunal correctionnel d'Alost, Étienne Martin Chompré, de lui fournir des renseignements exacts «sur [la] moralité et sur [la] conduite politique » des juges de son arrondissement afin de déterminer si l'article 26 de la loi du 19 fructidor an $\mathrm{V}$ était 《susceptible de recevoir son exécution » $\underline{74}$. Ancien président du club des jacobins de Marseille, Chompré avait été élu greffier du tribunal révolutionnaire et emprisonné après thermidor comme terroriste. Au moment de la crise des prêtres insermentés, il était par conséquent l'un des soutiens les plus fervents du Directoire. Il exprima à plusieurs reprises son aversion vis-à-vis du « fanatisme des Belges : l'esprit est si mauvais au reste ici, les opinions tellement fanatisées que le peuple attend à chaque instant l'Empereur dans la Belgique et qu'il croit à ces sourds instigateurs du 
désordre qui lui prêchent la désobéissance aux lois françaises et la haine contre le gouvernement et ses agents. Les Français, croyez-le, sont ici détestés et je dirais proscrits. Dès qu'ils veulent l'exécution des lois, ce sont des terroristes, des jacobins, des buveurs de sang, etc. C'est ainsi que nous sommes dépeints et montrés du doigt $\gg$ 75. À la même époque, son opinion à l'égard des juges était tout aussi négative : « je ne cesse de le dire, les Français sont exécrés dans ces départements. On entretient cet esprit et quelque tempérament que nous prenions, ces juges du Pays n'aiment ni ne souffrent notre gouvernement. Ils ont leur système d'indépendance et ils soutiennent avec la Constitution même qui est leur arme du moment pour la détruire s'ils peuvent $» \underline{76}$.

En dépit de ses nombreuses critiques et d'un certain lyrisme, Chompré se montra plus circonspect lorsque sa responsabilité fut directement engagée par l'acquittement du prêtre Vanransberghe. En réponse aux demandes de renseignement de Lambrechts, il minimisa la faute de Vandeputte, « un homme de mœurs douces mais faible de caractère, peu moyenné, et tenant à son poste par besoin ». L'explication donnée par Chompré résume le dilemme des magistrats rencontré constamment au cours de notre étude : « il voudrait plaire au gouvernement et ne pas déplaire à ses collègues et aux singuliers patriotes de cette commune, qui ne veulent des lois de la République que celles qui ne contrarient pas leurs opinions et leur système d'éloignement de notre constitution $» \underline{77}$. L'échange de courriers entre Lambrechts et Chompré produisit cependant l'effet voulu. Les 29 frimaire et 11 nivôse an VI (19 et 31 décembre 1797), le tribunal correctionnel d'Alost prononça la condamnation de quatre prêtres. Au cours des mois suivants, l'attitude équivoque de Vandeputte dans l'affaire Vanransberghe ne porta toutefois pas préjudice à sa carrière. Ancien avocat au Conseil de Flandre, nommé juge le 20 nivôse an IV (10 janvier 1796), il resta en fonction jusqu'à la fin du Directoire, à l'instar de soixante-et-un pourcents des juges élus en l'an V묘. Cette permanence fait écho au parcours d'autres juges rencontrés précédemment tels Lemoine, Ippersiel et Perlau. Une telle stabilité s'explique par les solidarités établies au sein de l'arrondissement entre les magistrats, qu'ils soient belges ou français, commissaires du gouvernement ou directeurs du jury. La solidité des relations et de l'entente était certainement aléatoire mais elle permit à nombre de magistrats de surmonter les orages de la Révolution.

\section{Notes}

1 Cette publication a été écrite dans le cadre du PAI 7/22 «Justice \& Populations : The Belgian Experience in International Perspective, 1795-2015».

$\underline{2}$ L'acculturation des modèles policiers et judiciaires français en Belgique et aux Pays-Bas (1795-1815), éd. E. BERGER, Bruxelles, Archives générales du Royaume, 2010.

3 Révolutions et justice pénale en Europe. Modèles français et traditions nationales (1730-1830), éd. X. Rousseaux, M.-S. Dupont-Bouchat et C. VaeL, Paris, L’Harmattan, 1999.

4 F. Stevens, Het tribunal criminel te Antwerpen (19 september 1794 - 19 februari 1795). Een nieuwe «Bloed Raed » op het einde van de $18^{e}$ eeuw ?, dans Acta Falconis, 1983, n 83, p. 176-221.

5 A. TiXHON, L'activité du tribunal correctionnel de Namur durant la période française (an IV1814), dans Annales de la société archéologique de Namur, t. 72, 1998, p. 291-341.

6 J. LogIE, Les magistrats des cours et des tribunaux en Belgique (1794-1814), Genève, Librairie Droz S.A., 1998. 
Résister ou collaborer ? Étude socioprofessionnelle des magistrats « belges »...

7 X. ROUSSEAUX et J.-P. NANDRIN, Le personnel judiciaire en Belgique à travers les révolutions (1780-1832). Quelques hypothèses de recherches et premiers résultats, dans Le personnel politique dans la transition de l'Ancien Régime au Nouveau Régime en Belgique (1780-1830), Courtrai, Anciens Pays et Assemblées d’État, 1993, p. 13-69.

$\underline{8}$ E. BERger, La justice pénale sous la Révolution. Les enjeux d'un modèle judiciaire libéral, Rennes, Presses universitaires de Rennes, 2008.

$\underline{9}$ Voir la contribution d'Aurore François et de Françoise Muller dans le présent numéro.

10 J. LoGIE, Les magistrats des cours et tribunaux en Belgique (1794-1814). Essai d'approche politique et sociologique, thèse de doctorat de l'université Paris IV, Index prosopographique, 1995 (cité après J. LoGIE, op. cit., index prosopographique). Les données ont été encodées sous la direction de Catherine Goffin (C. Goffin, Les magistrats dans les départements réunis sous le Directoire (an IV-an VIII) : Un corps social à part entière?, dans L'acculturation des modèles policiers et judiciaires français en Belgique et aux Pays-Bas (1795-1815), éd. E. BERGER, Bruxelles, Archives générales du Royaume, 2010, p. 99-108).

11 E. BERger, La justice pénale..., op. cit., p. 24-27.

12 Ibid.

1358 \% des juges refusèrent leur nomination (J. LogIE, op. cit., p. 117).

14 Ibid., p. 172.

15 Ibid., p. 111.

16 Ibid., p. 219.

17 Les données statistiques que nous présentons ont été produites à partir de la base des données prosopographiques des magistrats et des archives des directeurs du jury. Ces dernières sont conservées dans les fonds des tribunaux de première instance des Archives de l'Etat. La difficulté de la présente étude réside dans le fait que les données nécessaires à l'analyse des trajectoires socio-professionnelles des directeurs du jury ont été très mal conservées. Sur les trente-neuf arrondissements judiciaires créés à l'époque française, seuls dix présentent des archives exploitables. D'autre part, la rotation semestrielle des directeurs du jury accroit la difficulté à identifier les magistrats qui occupent cette fonction et à étudier leur activité au cours de cette période.

18 Soit du 28 novembre 1795 au 9 novembre 1799.

19 Seize sur nonante-quatre directeurs du jury.

20 Dix sur vingt-sept commissaires du gouvernement.

21 J. LogIE, op. cit., p. 236.

22 Ibid., p. 143-144.

$\underline{23}$ Ibid., index prosopographique, p. 339-340. 
24 Le prénom de Desmyter n'est pas connu.

$\underline{25}$ Les autres juges sont Edem Bourdault (dpt. Haute-Marne) dans l'arrondissement d'Anvers, Jean-René Maloigne (dpt. Seine) dans l'arrondissement de Nivelles, Pierre-Alexandre Lefèbvre (dpt. Somme) dans l'arrondissement de Bruxelles intérieur et Étienne Arbeltier (dpt. HauteMarne) dans l'arrondissement de Malines.

26 Les trois commissaires près les tribunaux correctionnels originaires d'autres départements sont : Étienne Martin Chompré (né à Paris) commissaire à Mons puis à Alost, Jean Antoine Chaix (né à Genève puis homme de loi à Paris) commissaire à Mons et Antoine Jean Bazire Lacoudraye (dpt. Vienne) commissaire à Gand.

27 J. LOGIE, op. cit., index prosopographique, p. 40-41.

$\underline{28} 175$ juges sur 202 .

29118 juges sur 202 .

30 J. LOGIE, op. cit., p. 236.

31 Cinquante-huit des septante directeurs du jury dont nous avons pu déterminer le lieu de résidence.

32 Quarante-cinq des septante directeurs du jury dont nous avons pu déterminer le lieu de résidence.

33 Trente-six des quarante-deux directeurs du jury dont nous avons pu déterminer le lieu de résidence.

$\underline{34}$ Huit des seize directeurs du jury dont nous avons pu déterminer le lieu de résidence.

35 Aucun des dix directeurs du jury dont nous avons pu déterminer le lieu de résidence.

36 J. LoGIE, op. cit., p. 237.

37 Les circulaires du ministre de la Justice sont accessibles sur le site suivant : http://www.digithemis.be/index.php/ressources/legislation\#circulaires.

38 Dix-neuf des vingt-trois commissaires du gouvernement près les tribunaux correctionnels dont nous avons pu déterminer le lieu de naissance.

39 Treize des vingt-trois commissaires du gouvernement près les tribunaux correctionnels dont nous avons pu déterminer le lieu de naissance.

40 Notons que deux de ces commissaires sont français : Jean Jacques Bernard Laroche (arr. Louvain, dpt. Dyle) et Étienne Martin Chompré (arr. Alost, dpt. Escaut).

41 Art. 644 du code des délits et des peines : «sont coupables de forfaiture (...) : $4^{\circ}$. Tout juge qui s'immiscerait dans l'exercice du pouvoir législatif, en faisant des règlements, ou qui se permettrait d'arrêter ou de suspendre l'exécution de la loi dans l'étendue de sa juridiction ».

$\underline{42}$ L'affaire De Hase et la problématique des prêtres insermentés ont fait l'objet de nombreux 
Résister ou collaborer ? Étude socioprofessionnelle des magistrats « belges »...

travaux. Pour une orientation bibliographique, voir : E. BERGER, La justice républicaine face aux prêtres insermentés sous le Directoire. Le cas des 9 départements belges, dans Justice et religion. Regards croisés : histoire et droit, éd. E. WENZEL, Avignon, Éditions universitaires d'Avignon, 2010, p. 57-69 ; J.-L. HALPÉRIN, Cassation et dénonciation pour forfaiture dans les départements réunis sous le Directoire, dans Justice et institutions françaises en Belgique (1795/1815). Traditions et innovations autour de l'annexion. Actes du colloque tenu à l'université de Lille II les 1, 2 et 3 juin 1995, éd. R. MARTINAGE et J.-P. Royer, Lille, L'Espace juridique, 1996, p. 245-258.

43 J. LogIE, op. cit., index prosopographique, p. 234.

44 Ibid, p. 204.

45 À l'exception du département des Deux-Nèthes peuplé de magistrats jacobins, les autres départements belges étaient composés majoritairement de conservateurs et de républicains modérés (Ibid., p. 239).

46 Ibid., p. 284-285.

47 Lettre adressée au juge de paix du canton d'Ath en date du 25 prairial an V (Archives de l'État (cité après $\mathrm{AE}$ ) à Mons, fonds du tribunal correctionnel de Mons, registre de correspondances du directeur de jury de Mons $n^{\circ} 8$, lettre $n^{\circ}$ A237).

48 Lettre adressée au ministre de la Justice Merlin en date du 12 fructidor an V (Archives nationales (cité après AN) BB18 400/DD4218).

49 Lettre adressée à l'accusateur public Joseph Giraud en date du 27 prairial an V (AE Mons, fonds du tribunal correctionnel de Mons, registre de correspondances du directeur de jury de Mons $n^{\circ}$ 8, lettre $n^{\circ}$ A240).

$\underline{50}$ L'atmosphère insurrectionnelle provoquée par la loi du 7 vendémiaire an IV est clairement décrite par l'accusateur public Giraud à Merlin dans son courrier du 28 prairial an V (16 juin 1797) : «Citoyen Ministre, le silence impolitique qu'on garde sur le sort de la loi du 7 vendémiaire relative à la déclaration des ministres du culte donne à ceux-ci l'audace de la révolte et aux agents du gouvernement et fonctionnaires publics le découragement de l'incertitude. La majorité est ou traitre à ses devoirs ou exposée aux insultes, aux violences et aux poignards du parti insurgé contre la loi et le Directoire. Un attroupement s'est montré dans la commune d’Enghien. Il a forcé l'adjoint à conscrire une permission de dire la messe. Il a jeté des cris de mort contre les autorités. Une émeute portant le même esprit s'est déclarée à Thuin lorsque les gendarmes constituaient prisonniers deux ministres du culte insoumis. On est obligé de faire marcher aujourd'hui une force militaire pour appuyer l'exécution de la loi. A Braine-le-Comte, on placarde le signalement des fonctionnaires publics, les vouant au massacre. La pièce ci-jointe porte la preuve avec elle. Dans notre commune, sous les yeux des autorités supérieures, on a tenté hier de braver la force publique. Les croyants se sont assemblés dans l'église de Saint-Germain. Un ministre du culte, sans avoir satisfait à la loi, a célébré son mystère. On a donné au prêtre insoumis le temps de se rendre chez lui. Un mandat d'amener l'a fait comparaître devant le juge de paix. Pendant sa traduction, un rassemblement a fait mine de vouloir le soustraire. Les gendarmes se sont montrés. Il n'y a eu que des propos de vitupération. Cependant, les rapports de la police ont fait juger indispensables des mesures militaires. Pour ma part, j'ai donné mes ordres à la gendarmerie » (AN BB18 400/DD4218). 
51 Le 12 fructidor an V (29 août 1797), le commissaire près le tribunal civil du département de Jemappes, Simon Nazaire Chenard écrit à Merlin : « le directeur, dit-on, donnera plutôt sa démission que d'ordonner la mise en jugement de ces vils cafards. Je viens d'en parler sérieusement à l'accusateur public qui ne me paraît pas disposé à agir à l'égard du directeur conformément à l'art. 287 du code des délits et des peines parce qu'il craint que le tribunal ne fasse rien que d'insignifiant à ce sujet et qu'au reste ce directeur ne donnât sa démission. Je ne vois, moi, aucun inconvénient à ce qu'un magistrat qui ne veut pas exécuter la loi quitte son poste, c'est au contraire un bien »(AN BB18 400/DD4218).

52 Circulaire adressée aux juges de paix de l'arrondissement de Malines en date du 3 prairial an V (AN BB18 563/DD 3929).

53 Lettre adressée au ministre de la Justice en date du 15 prairial an V (AN BB18 563/DD3929).

54 Lettre adressée au commissaire du gouvernement près le tribunal correctionnel de Malines, Sayavedra, en date du 7 messidor an V (AN BB18 563/DD3929).

55 Lettre adressée au ministre de la Justice en date du 14 prairial an V (AN BB18 284/DD 3877).

56 Ancien président du tribunal criminel de la Dyle, Bonaventure avait été élu au Conseil des Cinq-Cents. Il prit en prairial publiquement position en faveur de la suspension des articles litigieux de la loi du 7 vendémiaire an IV.

57 Lettre adressée au ministre de la Justice en date du 5 messidor an V (Archives de l'État à Mons, fonds du tribunal correctionnel de Mons, registre de correspondances du commissaire du

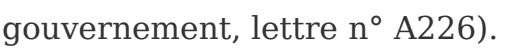

58 Lettre adressée au Directoire en date du 15 prairial an V (AN BB18 563/DD 3929).

$\underline{59}$ J. Logie, op. cit., index prosopographique, p. 119-120 et 369-370.

60 Ibid., p. 315.

61 Lettre adressée au ministre de la Justice en date du 14 prairial an V (AN BB18 284/DD 3877).

62 Lettre adressée à son collègue près le tribunal correctionnel de Tournai, en date du 14 fructidor an V (AE Mons, fonds du tribunal correctionnel de Mons, registre de correspondances du commissaire du gouvernement, lettre $\mathrm{n}^{\circ} \mathrm{A} 263$ ).

$\underline{63}$ Voir note 45.

64 Lettre adressée au ministre de la Justice en date du 23 thermidor an V (AN BB18 400/DD 4375).

65 J. LOGIE, op. cit., index prosopographique, p. 254.

66 Lettre adressée au ministre de la Justice en date du 14 fructidor an V (AN BB18 400/DD 5190).

67 La correspondance de Lacoudraye témoigne à plusieurs reprises du souci de ce dernier de gagner l'opinion publique : "j'ai répandu et accrédité par tous les moyens qui ont dépendu de moi les écrits que j'ai cru les plus propres à ramener l'opinion publique et à faire apprécier les 
Résister ou collaborer ? Étude socioprofessionnelle des magistrats « belges »...

principes établis par le Directoire exécutif» (Lettre adressée au ministre de la Justice en date du 23 thermidor an V).

68 E. BERGER, La justice pénale..., op. cit., p. 70.

69 Le 7 pluviôse an VI (26 janvier 1798), le tribunal criminel du département de l'Escaut condamna De Hase à trois mois d'emprisonnement et à 500 livres d'amende (Archives de l'État à Gand, Cour d'assises de Flandre occidentale, registre aux jugements $n^{\circ} 14$ ).

$\underline{70}$ Quarante-deux affaires sur un total de septante-huit.

71 Seize affaires sur un total de septante-huit.

$\underline{72}$ L'augmentation des jugements en l'an VI est également due à la mise en jugement d'affaires instruites en l'an $\mathrm{V}$ et dont le renvoi devant la justice correctionnelle avait été retardé dans l'attente d'une réforme de la législation relative à la police des cultes.

73 Lettre adressée au commissaire près le tribunal criminel du département de l'Escaut, Lacoudraye, en date du 12 frimaire an VI (AN BB18 294/DD 2774).

74 Lettre adressée au commissaire près le tribunal correctionnel de l'arrondissement d'Alost, Chompré, en date du 12 frimaire an VI (AN BB18 294/DD 2774).

$\underline{75}$ Lettre adressée au ministre de la justice en date du 4 thermidor an V (AN BB18 294/DD 2774).

76 Lettre adressée au ministre de la justice en date du 28 prairial an V (AN BB18 294/DD 2774).

77 Lettre adressée au ministre de la Justice en date du 21 frimaire an VI (AN BB18 476/DD 7419).

78 Tout comme Lemoine, Ippersiel et Perlau, la carrière de Vandeputte se prolongea bien au-delà de la chute du Directoire. Devenu juge au tribunal de Gand en l'an VIII, il fut maintenu lors de l'épuration de 1811 et sous Guillaume I ${ }^{\mathrm{er}}$. Vandeputte décéda en fonction en 1825 (J. LoGIE, op. cit., index prosopographique, p. 365).

PDF généré automatiquement le 2020-07-12 07:27:10

Url de l'article : https://popups.uliege.be:443/1370-2262/index.php?id=365 